Supporting Information

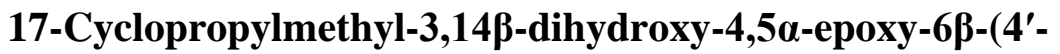

pyridylcarboxamido)morphinan (NAP) modulating the mu opioid receptor in a

\section{biased fashion}

Yan Zhang, ${ }^{*, \dagger}$ Dwight A. Williams, ${ }^{\dagger \neq}$ John M. Streicher, ${ }^{\S}$ Yunyun Yuan, ${ }^{\dagger}$ Saheem A.

Zaidi, $^{\dagger}$ Amanda Braithwaite, ${ }^{\S}$ Hamid I. Akbarali, ${ }^{\ddagger}$ William L. Dewey, ${ }^{\ddagger}$ Dana E. Selley, ${ }^{\ddagger}$

Edward J. Bilsky

${ }^{\dagger}$ Department of Medicinal Chemistry, Virginia Commonwealth University, 800 East

Leigh Street, Richmond, VA 23298, USA

‡Department of Pharmacology and Toxicology, Virginia Commonwealth University, 410

North 12th Street, Richmond, VA 23298, USA

${ }^{\S}$ Department of Biomedical Sciences, College of Osteopathic Medicine, University of New England, 11 Hills Beach Road, Biddeford, ME 04005, USA 
Figure 1S. $\mathrm{Ca}^{2+}$ flux assay in $\mathrm{G}_{\alpha q i 5}$ transfected hMOR-CHO cells. The results shown are representative of at least three independent experiments. The detection time was extended to $10 \mathrm{~min}$ in contrast to $2 \mathrm{~min}$.

\section{Time Course Study (10 min)}

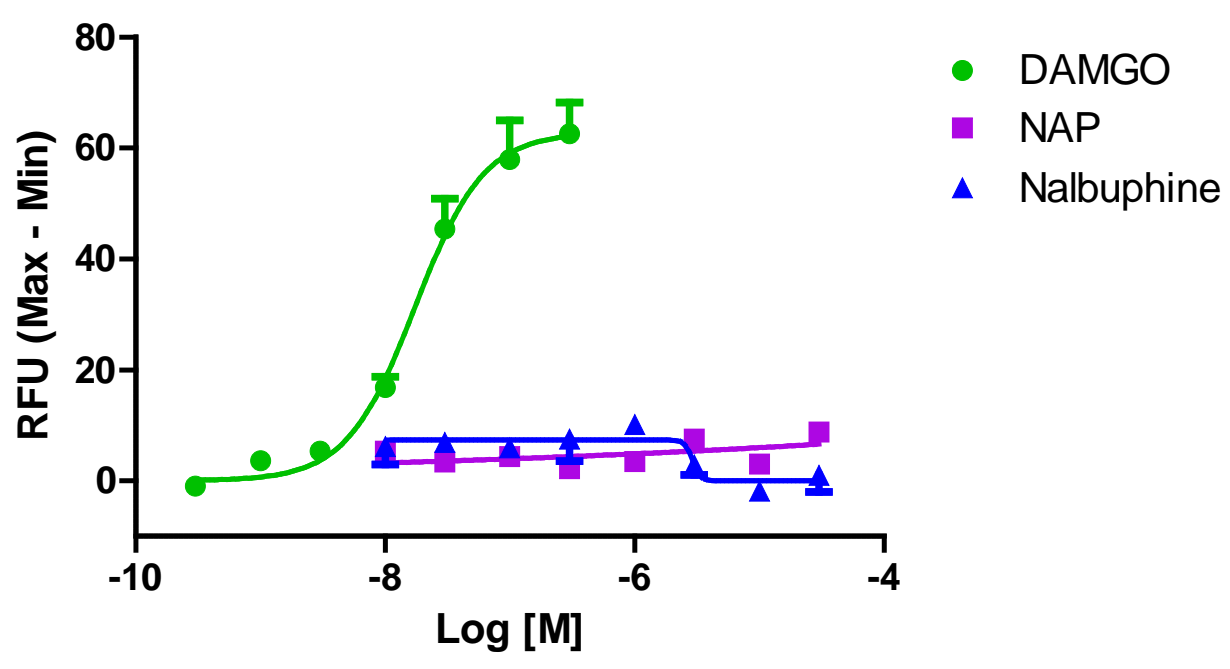

